\title{
La nueva Etapa del "Desarrollo Constructivo" de las relaciones sino-latinoamericanas ${ }^{1}$
}

\section{The new "Constructive Development" Stage of Sino-Latin American Relations}

\author{
Chen, Yuanting y Li, Han $^{2}$
}

Resumen: Después de experimentar las etapas del desarrollo espontáneo y del desarrollo independiente, las relaciones sino-latinoamericanas han entrado en una nueva fase del "desarrollo constructivo" que se caracteriza por el objetivo estratégico de construir una nueva estructura y una comunidad de destino común, un con un nuevo marco de cooperación basado en la negociación y en la ganancia mutua. Como resultado de esto, los lazos entre China y América Latina han conseguido muchos avances en los terrenos político, económico, cultural y de la cooperación multilateral. Sin embargo, debido a la incertidumbre del mundo y las transformaciones de las dos partes, la cooperación entre ambos lados sigue enfrentando muchos desafíos. En la actualidad, hay varias contradicciones importantes que deben ser resueltas: la primera, es la brecha entre el mejoramiento de mecanismos de cooperación y sus expectativas; la segunda, es la cada vez más fuerte presencia estratégica de China en América Latina y la capacidad de aceptación de este continente. La última es la necesidad urgente de desarrollar y profundizar esta relación tras la falta del conocimiento mutuo.

Palabras claves: relaciones sino-latinoamericanas; desarrollo constructivo; comunidad de destino común; nueva etapa.

Abstract: After experiencing the stages of spontaneous development and independent development, Sino-Latin American relations have entered a new phase of "constructive development" characterized by the strategic objective of building a new structure and a community of common destiny, by the Strategic route of establishing a new framework of cooperation, and by the principle of cooperation with negotiation, mutual gain, and not against the third. As a result, the ties between China and Latin America have made much progress in the political, economic, cultural and multilateral fields. However, due to the uncertainty of the world and the transformations of the two regions, cooperation between the two sides continues to face many challenges. At present, there are several important contradictions that need to be resolved: the first is the gap between improving cooperation mechanisms and their expectations; The second is China's increasingly strong strategic presence in Latin America and the continent's capacity for acceptance. The latter is the urgent need to develop this relationship and the lack of mutual knowledge.

Key words: Sino-Latin American relations; constructive development; common destiny community; new stage

1 Recibido: 01/08/2017. Aceptado: 25/10/2017

2 La Doctora Chen Yuanting es investigadora asociada del Instituto de América Latina de la Academia China de Ciencias Sociales (ILAS-CASS); la Doctora Li Han es investigadora asistente del Instituto de América Latina de la Academia China de Ciencias Sociales (ILAS-CASS). 


\section{Introducción}

A partir del AÑO 2015, "la teoría de la nueva etapa" se ha convertido en una nueva e importante perspectiva para analizar la relación entre China y América Latina y el Caribe $(A L C)$. Algunos académicos chinos sostienen que en julio de 2014 , la visita del presidente Xi Jinping a cuatro países latinoamericanos significó que los lazos entre ambos lados entraran en "una nueva etapa de desarrollo integral". Las iniciativas y la estructura de cooperación propuestas por el presidente chino no sólo explican los objetivos, sino también plantean una hoja de ruta detallada (Shuangrong, 2016). Otro grupo de académicos estiman que la celebración de la Primera Reunión Ministerial del Foro China-CELAC significó la renovación de la relación (Hongying, 2015) y la aplicación de un nuevo modelo de cooperación a nivel regional y omnidireccional, que significa la cobertura completa de los mecanismos de cooperación integral entre China y los países en vías de desarrollo. Se evidencia la importancia de esta región en el panorama de la diplomacia china (Dingli, 2016). Además, otros académicos expresan que la visita del líder chino por tercera vez a este continente en 2016, y el Segundo Documento sobre la Política de China hacia América Latina se consideran como un signo de que la vinculación sino-latinoamericana está evolucionando cada vez más (Baiyi, 2015).

Los analistas y organismos internacionales también creen que los lazos entre ambas partes han entrado en una nueva etapa de desarrollo. Por su parte, Enrique Dussel Peters, coordinador del Centro de Estudios China-México de la Universidad Nacional Autónoma de México, sostiene que después de ingresar en el siglo XXI, la relación entre China y ALC podría dividirse en dos etapas: la primera, se caracteriza por el auge del comercio y la inversión; la segunda se muestra por el intercambio cultural, el aumento de los inmigrantes chinos en América Latina, el dinamismo del turismo y el establecimiento del mecanismo de diálogo interregional (Dussel Peters, 2015). Un informe emitido por parte de la Organización para la Cooperación y el Desarrollo Económicos, el Banco Interamericano de Desarrollo y la Comisión Económica para América Latina, describen que la transformación y la transferencia de riqueza encabezadas por parte de China tienen una influencia global. China y América Latina deberían trabajar juntos para profundizar las relaciones de cooperación estratégica (Latin American Economic Outlook, 2016). Algunos académicos creen que las relaciones de China con muchos países en desarrollo, han atravesado un período de "luna de miel" y ahora es necesario establecer una relación más madura y compleja (Maoxiu, s/f).

A pesar de algunas diferencias entre estas opiniones, se observa un punto común, todos concuerdan en que los vínculos sino-latinoamericanos han entrado en una nueva etapa de desarrollo. Este artículo trata de analizar los lazos desde la perspectiva de las transformaciones de estrategias diplomáticas de ambas partes, y propone que, después de experimentar las etapas del desarrollo espontáneo e independiente, las relaciones sino-latinoamericanas han entrado en una nueva fase de "desarrollo constructivo". En este sentido, nos formulamos las siguientes interrogantes: ¿cómo se define este nuevo modelo de desarrollo, cuales son sus desafíos y oportunidades de futuro? 


\section{Implicaciones de la nueva etapa}

\section{a. Retrospectiva de las relaciones sino-latinoamericanas: desde el desarrollo espontáneo al independiente}

Desde la fundación de la República Popular de China en el año 1949, las relaciones sino - latinoamericanas tienen una historia de casi 70 años. Durante este período, debido a los cambios del contexto internacional y de estrategias exteriores de ambas partes, los lazos entre China y América Latina han experimentado una transformación en términos de impulso, orientación y logros, y se han ido conformando en diferentes etapas.

Del año 1949 a los ochentas años del siglo XX, los vínculos entre ambas partes estaban en una fase de desarrollo espontáneo. Durante este período, China y América Latina pertenecían a los dos bloques enfrentados durante la Guerra Fría, cuyas estrategias exteriores estaban influenciadas fuertemente por la ideología. De manera tal que el entorno internacional y las situaciones políticas internas no favorecieron el desarrollo de una relación más cercana. Además, debido a la distancia geográfica y la falta de entendimiento, algunos gobiernos latinoamericanos incluso tomaron una actitud hostil hacia China por los factores ideológicos. Esto llevó a la carencia de voluntad para el acercamiento entre ambas partes. En aquel entonces, la Unión Soviética y los Estados Unidos ocuparon un lugar prioritario en las políticas exteriores tanto de China como de los países latinoamericanos, y la relación entre las dos partes se situaba en una posición marginal, subordinada a sus lazos con los dos gigantes. Por eso, China y ALC carecían de la capacidad de promover una vinculación de forma más independiente y autónoma, lo que mantenía un desarrollo muy lento y el principal avance fue entonces la normalización de las relaciones bilaterales.

Desde finales de la década de 1980 hasta 2012, antes del XVIII Congreso Nacional del Partido Comunista de China, las relaciones sino-latinoamericanas entraron en una etapa de desarrollo independiente. En este período, la Guerra Fría había terminado, y el entorno internacional se caracterizó por la multipolarización política, la globalización económica y el ascenso de un grupo de países emergentes. China y América Latina dedicaron sus esfuerzos a desarrollarse y establecer un nuevo orden político y económico internacional, diversificando sus diplomacias para fortalecer las relaciones y la cooperación con los países en desarrollo. En este contexto, se incrementó la importancia estratégica de China y América Latina, la voluntad de cooperación y el impulso de sus lazos. Por eso, los vínculos entre ambos lados eran más independientes que antes. Por ejemplo, a pesar de las sanciones económicas y el aislamiento diplomático de Estados Unidos y otros países occidentales, China priorizó sus relaciones con los países latinoamericanos, consiguió sus apoyos y la invitación de cinco países del continente para hacer una visita oficial. Al ingresar en el nuevo siglo, con el rápido desarrollo de la economía de China, las diversas fuerzas políticas latinoamericanas -no sólo de la izquierda- consideran al país asiático como su principal socio, y dan mayor importancia a la vinculación bilateral. Gracias a la creciente voluntad e independencia y también debido al rápido desarrollo de la economía de ambas partes y su ascenso en el marco internacional, las relaciones sinolatinoamericanas se desarrollan en forma multifacética, sostenida y profunda, sentando una base sólida para el establecimiento de la Asociación de Cooperación Integral. 


\section{b. Consensos e implicaciones.}

Después de las dos etapas de desarrollo, las relaciones sino-latinoamericanas han establecido una red de contacto más amplia, y mantienen una buena tendencia de desarrollo. En vista de la creciente importancia estratégica de tales relaciones, ambas partes desean promover sus vínculos a un nivel más alto. En este contexto, las relaciones sinolatinoamericanas han entrado en una nueva etapa de: "el desarrollo constructivo" que se refiere a la planificación estratégica de la vinculación por parte de los líderes en cuanto a los objetivos, la ruta y los principios de cooperación y otros aspectos.

El desarrollo de los vínculos sino-latinoamericanos se destaca por el "postdesarrollo", lo que ha significado su retraso en comparación con las relaciones de China con los países grandes, los vecinos, e incluso con los países africanos. Una de las razones más importantes es que la distancia geográfica entre China y América Latina, en cierta medida, obstaculiza el desarrollo de las relaciones bilaterales, pero por otro lado, favorece mantener la paz que se sirve como la base para impulsar la amistad y la igualdad entre ambas partes, lo que contribuye la entrada en una nueva fase del desarrollo. La otra razón es que el "post-desarrollo" permite el escalonamiento de tales relaciones para alcanzar una nueva etapa diseñada por las dos partes.

Después de las dos etapas de desarrollo mencionadas (reforma y apertura), China ha mantenido un alto crecimiento por más de 30 años, y se ha convertido en la primera potencia comercial y la segunda economía más grande del mundo, ejerciendo una influencia cada vez más importante en los asuntos internacionales. El creciente poder nacional de este país asiático contribuye a la expansión de sus intereses, el cambio de su status internacional y la necesidad de transformar su papel. A partir de finales de 2012, un nuevo colectivo de liderazgo de China planteó el sueño chino, es decir "la gran revitalización de la nación china" e inició "un nuevo camino de la diplomacia con característica de China" (Jinping, 2016:260) que se manifestó en varios aspectos: en primero lugar, la transformación de la diplomacia "reactiva" hacia la "planeación activa" (Jiemian, 2015), con una altura estratégica. El segundo es la construcción de una "cooperación de ganarganar" como el núcleo de las nuevas relaciones internacionales, con el fin de crear "la comunidad de destino humano" y "la comunidad de destino regional", entre otros. En tercer lugar, China despliega una diplomacia omnidireccional y multifacética. Además, China también presentó su nueva visión sobre Rectitud y Beneficio que es "desarrollar lo político y lo económico simultáneamente, mantener el equilibrio entre el interés y la moral y, a veces incluso poner la moral antes del interés" (Jiemian, 2014: 10). Por el último, se enfatiza la diplomacia cultural y humanística, con el objetivo de transmitir el valor de que se busca por parte de China, y asociar "el sueño chino" con los sueños de todos los pueblos del mundo.

China percibe a América Latina como la fuerza constructiva para establecer nuevo modelo de relaciones internacionales. En primer lugar, esta región sirve como una base principal para la realización de la asociación estratégica en la diplomacia de China. Desde comienzos de la década de los noventa, China ha desplegado "asociaciones estratégicas" con seis países latinoamericanos: Brasil, Venezuela, México, Argentina, Perú y Chile. Posteriormente, China decidió elevar el estatus de estas relaciones al de "asociación estratégica integral". 
En segundo lugar, América Latina juega un papel clave para un modelo de cooperación Sur-Sur y tiene el peso creciente en la acción diplomática de China que se basa en la igualdad, reciprocidad integral y beneficio mutuo.

Por último, América Latina y China aspiran al desarrollo pacífico y un nuevo modelo de relaciones internacionales basado en la paz, el desarrollo, la cooperación y la ganancia mutua; por lo tanto, esta región es una de las prioridades de la estrategia del "desarrollo constructivo" impulsada por el gobierno chino.

Las naciones latinoamericanas han venido reconociendo este nuevo modelo y desean profundizar sus vínculos con China. En la actualidad, estos países afrontan las siguientes dificultades: falta una estrategia clara hacia China, ya sea individualmente o en conjunto y al mismo tiempo, existe heterogeneidad en el continente en cuanto al deseo de impulsar lazos con el país asiático. Debido a la competitividad y el desequilibrio en las relaciones comerciales, los grupos económicos en México por ejemplo, dan menor importancia a la vinculación con China que el propio gobierno de Enrique Peña Nieto. Por otro lado, los cambios de gobierno ocurridos en Argentina y Brasil causaron un ajuste moderado en sus políticas exteriores, pero el resultado es que los dos países tratan de reforzar sus vínculos de forma integral con China. Las naciones que han establecido el Tratado de Libre Comercio con China como Chile y Perú dedican muchos esfuerzos para profundizar las relaciones bilaterales. Esto denota que a los países latinoamericanos les falta capacidad suficiente para promover su propia estrategia exterior. Asimismo, y como resultado de las caídas de los precios de los productos básicos a nivel mundial, las economías de esta región registraron un proceso de desaceleración. Durante 2015 y 2016, este continente atravesó el crecimiento negativo. La inestabilidad política agravó la situación económica. Las naciones latinoamericanas desean diversificar sus estrategias exteriores para mejorar las economías y siendo China, la segunda economía más grande del mundo, emerge como una opción importante para América Latina. Por lo tanto, podríamos afirmar que China desempeña un papel activo y de promotor en las relaciones bilaterales.

Los países de América Latina y el Caribe han atravesado un rápido crecimiento en el siglo XXI y han desempeñado un papel cada vez más importante en los asuntos internacionales. Sin embargo, después de la crisis financiera mundial en 2008 , como consecuencia de la debilidad de la economía mundial, la caída de los precios internacionales de los productos básicos y la contracción del comercio internacional, la economía de América Latina ha sufrido fuertes pérdidas que se reflejan en el lento crecimiento que ha experimentado desde 2012, e incluso un crecimiento negativo en 2015 y 2016. Con el fin de estimular el crecimiento y promover la reforma económica, las naciones de Latinoamérica y el Caribe han adoptado formas de diplomacia más pragmáticas y han puesto énfasis en la diplomacia económica. Además, con la reducción de la influencia de Estados Unidos en América Latina y la transferencia del peso del crecimiento mundial, la diplomacia de esta región continúa diversificándose, prestando más atención a Asia y la región del Pacífico. El fortalecimiento de las relaciones con China se ha convertido en un consenso regional en América Latina con el objetivo de ampliar la cooperación económica y comercial o de diversificarla.

Después de estas dos etapas de desarrollo, las relaciones sino-latinoamericanas han establecido una red de contacto más amplia, y mantienen una buena tendencia de desarrollo. China se ha convertido en el segundo mayor socio comercial de América Latina, y América Latina se ha convertido en el segundo destino de la inversión de China. La cooperación en el campo de la cultura, la ciencia y la tecnología también ha obtenido muchos progresos. 
En vista de la creciente importancia estratégica de tales relaciones, ambas partes desean promover sus vínculos a un nivel más alto. En este contexto, las relaciones sinolatinoamericanas han entrado en una nueva etapa de "desarrollo constructivo" que se refiere a la planificación estratégica de la vinculación por parte de los líderes en cuanto a los objetivos, la trayectoria, los principios de cooperación y otros aspectos.

Objetivo Estratégico: construcción de una nueva estructura de relaciones sinolatinoamericanas y creación de la comunidad de destino común China-Latinoamérica

En las dos etapas anteriores, las relaciones entre China y América Latina se desarrollaron sin planificación clara y específica, lo que se puede definir como un modelo "reaccionario".

En 2014, el presidente chino Xi Jinping hizo su visita al continente latinoamericano y planteó la construcción de una nueva estructura de relaciones China-América Latina y el Caribe en el que se involucraron las cinco partes, es decir, la sinceridad y confianza mutua en lo político, la cooperación de ganancias compartidas en lo económico y comercial, el aprendizaje mutuo en lo cultural y humano, la estrecha colaboración y coordinación en los asuntos internacionales y la promoción mutua entre la cooperación en su conjunto y las relaciones bilaterales, a fin de crear una comunidad de destino compartido para avanzar juntos. Los países de América Latina y el Caribe dieron muestras contundentes a favor de las iniciativas planteadas por China. En este sentido, el Presidente de Uruguay, José Mujica, expuso que es un programa muy importante para la Cooperación China-América Latina y el Caribe. El concepto de "comunidad de destino común" significa que las relaciones China-América Latina y el Caribe se han desarrollado a un nivel más alto, y constituye un objetivo estratégico. Mientras tanto, los vínculos entre ambos lados se caracterizan por un modelo "activo y constructivo".

Ruta estratégica: establecimiento de un nuevo marco y modelo de cooperación integral

En enero de 2015, durante la Primera Reunión Ministerial del Foro China-CELAC, se estableció el programa de cooperación, con el fin de forjar la comunidad de destino común China-América Latina y el Caribe. Ambas partes elaboraron conjuntamente el "Programa de Cooperación China-América Latina y el Caribe para el Período 20152019", que sirve como guía para la cooperación del próximo lustro.

Se crea de esta manera un nuevo modelo de cooperación: la cooperación económica y comercial, que sirve de motor para promover los vínculos entre ambos lados. Para mantener el impulso, es necesario buscar nuevos espacios de desarrollo. Por lo tanto, durante la visita oficial del presidente chino Xi Jinping en América Latina en 2014, se propuso trabajar juntos para construir un nuevo marco de cooperación " $1+3+6$ ". El "1" se refiere a "un programa", es decir, elaborar el "Programa de Cooperación ChinaAmérica Latina y el Caribe para el Período 2015-2019", con la realización de un crecimiento inclusivo y el desarrollo sostenible como el objetivo. El " 3 " representa los "tres grandes motores": el comercio, la inversión y la cooperación financiera como promotores del desarrollo integral de la cooperación pragmática entre China y América Latina y el Caribe. El "6" implica las "seis áreas", es decir: energía y recursos naturales, construcción de infraestructuras, agricultura, manufactura, innovación científica y tecnológica y tecnología de información como áreas prioritarias de cooperación para promover el acoplamiento industrial entre China y América Latina y el Caribe. Los países de América Latina y el Caribe aprecian fuertemente las importantes iniciativas y medidas planteadas por Xi 
Jinping para reforzar la cooperación China-América Latina y el Caribe y apoyan al establecimiento del nuevo marco de cooperación.

El presidente de Ecuador Rafael Correa expresó que el esquema 1-3-6 es una medida pragmática y a la vez respetuosa de América Latina. En 2015, el primer ministro chino hizo una visita a esta región y explicó que China propone que ambas partes exploren un nuevo modelo de cooperación $3 \times 3$. Es decir, la construcción conjunta de las tres grandes vías: (i) logística, energética e informática, (ii) materializar la interacción virtuosa entre las empresas, la sociedad y el gobierno, y (iii) ampliar los tres canales de financiación (fondos, créditos y seguros). Todos los nuevos modelos de cooperación favorecen la profundización de las relaciones sino-latinoamericanas y el Caribe.

Principios de cooperación: negociación y ganancia mutua, no se apunta contra nadie

En primer lugar, tanto China como los países de América Latina son países en desarrollo, que se adhieren a los principios diplomáticos como la independencia, la autonomía, la paz y la diversidad, y abogan por establecer un orden político y económico internacional más justo al tiempo que consiguen materialización del desarrollo propio. En este sentido, las dos partes tienen muchos aspectos complementarios en cuanto a la etapa, la noción y el modelo de desarrollo. Por eso, "la asociación de cooperación integral ChinaALC está basada en la igualdad y el beneficio mutuo y, se dirige al desarrollo común, no se apunta contra nadie ni excluye a ninguna tercera parte" (Ministerio de Asuntos Exteriores (2016), Documento sobre la política de china hacia América Latina y el caribe. Disponible en: http://www.fmprc.gov.cn/esp/wjdt/wjzc/t1418256.shtml). En el "Programa de Cooperación China-América Latina y el Caribe para el Período 2015-2019", el decimocuarto punto es sobre la Implementación de Iniciativas y expresa:

“El presente Plan de Cooperación se implementará bajo los principios de flexibilidad y participación voluntaria, de conformidad con las políticas nacionales y las provisiones de los sistemas jurídicos nacionales de los respectivos países y, su implementación no afectará ningún programa de cooperación bilateral acordado por alguna de las Partes, ni sustituirá los acuerdos bilaterales, decisiones o compromisos establecidos entre las Partes".

Wang Yi, el Ministro de Relaciones Exteriores de China, señaló que la Cooperación China-América Latina y el Caribe es un nuevo modelo del desarrollo común y la cooperación Sur - Sur, lo que ponen en pleno juego las ventajas complementarias, consolidan constantemente la nueva estructura de cooperación de beneficio mutuo caracterizada por la diversidad abierta y la convergencia profunda, y no se apunta contra nadie ni excluye a ninguna tercera parte (Wang Yi, 2015, 《王毅: 中拉合作不针对、不排斥第三方 》, 新华网, La cooperación sino-latinoamericana no está dirigida ni excluye a otros, de xinhuanet. http://news.xinhuanet.com/world/2015-01/09/c_1113944290.htm, 访问日 期： 2017 年 7 月 1 日。) 


\section{3. Éxitos conseguidos en la nueva etapa}

\section{a. En el ámbito político}

En primer lugar, las relaciones sino-latinoamericanas en esta etapa se destacan por la diplomacia de cumbres. Despúes del XVIII Congreso Nacional del Partido Comunista de China (PCCh), el presidente Xi Jinping realizó tres viajes oficiales a esta región en el año 2013, 2014 y 2016. El primer ministro Li Keqiang hizo la primera visita en 2015. Todo esto demuestra que los líderes chinos ponen mucha atención a los lazos entre ambos lados. Para los países latinoamericanos, la importancia de China también ocupa un lugar importante en sus relaciones exteriores. Por ejemplo, desde el año 2013, los líderes argentinos tanto de la izquierda como de la derecha hicieron visitas al gigante asiático. El presidente Macri participó en la Cumbre del G-20 en 2016 y en el Foro de "una Franja y una Ruta" en 2017, y su predecesora Cristina Fernández, visitó Beijing en 2015. Todo esto refleja que los distintos grupos sociales y políticos en este continente reconocen la necesidad de profundizar su vínculación con esta potencia. Mientras tanto, los líderes de ambos territorios se reunieron en varias ocasiones para impulsar el entendimiento mutuo, a través de mecanismos multilaterales como la Organización de las Naciones Unidas (ONU), el Foro de Cooperación Económica Asia-Pacífico, los BRICS, el G-20, la Cumbre de Seguridad Nuclear, entre otros.

En segundo lugar, China y América Latina convocan encuentros a gran escala y de manera colectiva. En 2013, el mandatario chino Xi Jinping mantuvo conversaciones con sus homólogos de ocho países caribeños, y durante su segunda gira por la región en 2014, Xi Jinping mantuvo encuentros con los líderes y representantes de CELAC, y emitieron la Declaración Conjunta de la Cumbre de Brasilia de Líderes de China y de Países de América Latina y el Caribe. Eso evidencia la amplia cobertura geográfica de la diplomacia de cumbres de China. Además, la primera visita de un presidente chino a Trinidad y Tobago en 2013 tuvo una importancia histórica, lo que muestra una estrategia multidimensional de China. Los primeros ministros de Bahamas y Dominica sostuvieron que el gobierno de Beijing es un activo socio y su interés por el Caribe es muy constructivo.

Por último, China y América Latina están renovando sus relaciones asociativas, lo que forma parte de su diplomacia integral (Yi, 2017). En 2012, China inició con Brasil el paso de la "relación asociativa estratégica" a la "relación asociativa estratégica integral"; seguido por México y Perú en 2013. Un año después, avanzó con el calificativo "integral" hacia países como Argentina y Venezuela, mientras que en enero de 2015 se extendió a Ecuador y a Chile en 2016. Asimismo, mantiene relaciones cooperativas de alto nivel con Costa Rica y Uruguay. La decisión de elevar de status las relaciones con las prinicpales naciones es favorable para el impulso de lazos entre China y la región. El presidente mexicano, Enrique Peña Nieto, estimó que la asociación estratégica integral permite ofrecer estímulos comerciales, y buscar nuevos modos y terrenos asociativos, con el fin de dar mayor equilibrio a la balanza comercial y caminar hacia una sinergia de beneficios mutuos.

\section{b. En el ámbito económico}

La cooperación económica se caracteriza por la diversificación. El incremento de volumen comercial entre China y América Latina se ha desacelerado, pero la inversión de 
China en este continente mantiene su nivel de crecimiento de diversas maneras y en campos mucho más amplios. En 2015, la inversión directa no financiera de China en esta región sumó 21460 millones de dólares, registrando un aumento del 67\% para el 2016, las cifras fueron de 29800 millones de dólares y un incremento del 39\% respecto del año anterior. Además, la inversión China en la región mostró mayor diversidad en cuanto a sus campos, modos de cooperación y entidades de inversión. América Latina recibió financiamiento de China en una amplia gama de sectores que además de energía, minería e infraestructura, incluye agricultura, industria manufacturera, industria informática, sector de servicios, comercio electrónico y transporte aéreo, entre otros. Al mismo tiempo, las empresas chinas continúan fortaleciendo su cooperación con naciones latinoamericanas para poner en marcha proyectos de infraestructura con el tradicional modelo EPC (Ingeniería, Adquisición y Contratación) y han empezado a probar modelos como BOT (Crear, Operar y Transferir), para llevar a cabo la cooperación con la región latinoamericana. En el tema de cooperación de contratación para obras, el volumen de éstas contrataciones a empresas chinas en Latinoamérica llegó a 18160 millones de dólares y se llevó a cabo un volumen de 16400 millones de dólares en el 2015, lo que suponía un incremento de $10.3 \%$ y $24.4 \%$ respectivamente. Un año después, estas cifras alcanzaron 19120 y 16030 millones de dólares, con una tasa de crecimiento de $5.3 \%$ y $2.3 \%$ respectivamente. Las medidas de apoyo financiero han contribuido a la implementación de proyectos de gran envergadura. Durante 2016, se pusieron en marcha todas las medidas financieras anunciadas por dirigentes chinos durante sus visitas a América Latina. Dichas medidas han conseguido resultados muy positivos. Hasta la fecha, muchos de los proyectos contemplados en el Fondo de Cooperación China-América Latina, tales como préstamos preferenciales, y los préstamos especiales para la infraestructura entre China y América Latina, se han implementado de manera ordenada. Gracias al Fondo de Cooperación entre China y Brasil en el sector de la capacidad productiva, empresas chinas han logrado el derecho de la franquicia de dos centrales hidroeléctricas de la nación sudamericana. La puesta en marcha y su implementación de los proyectos referidos han contribuido de forma positiva a la cooperación entre las dos partes en la infraestructura, la exploración de energías y recursos, electricidad, agricultura, manufactura, equipos marinos y biotecnología, entre otros sectores ${ }^{3}$. A través del comercio y el intercambio por muchos años, los países latinoamericanos profundizan el deseo de acercarse a China ${ }^{4}$.

Se crean nuevos mecanismos de cooperación. En el 2014, el presidente chino propuso el lanzamiento de un fondo específico para financiar proyectos de infraestructura, llegando a 20 mil millones de dólares que comenzaba con capital inicial de 10 mil millones de dólares. China también planteó líneas de crédito en condiciones preferenciales por un monto de 10 mil millones de dólares para los países de la Comunidad de Estados Latinoamericanos y del Caribe, y se activó integralmente el Fondo de Cooperación ChinaAmérica Latina y el Caribe con una aportación comprometida de la parte china de 5 mil millones de dólares. China puso en marcha formalmente el Fondo Especial para la Coope-

3 http://www.mofcom.gov.cn/article/ae/ai/201702/20170202513555.shtml página web de Ministerio de Comercio, fecha de consulta:10 de abril de 2017.

4 A golden opportunity : China's president ventures into Donald Trump's backyard, https://www.economist.com/news/americas/21710307-chinas-president-ventures-donald-trumpsbackyard-golden-opportunity 
ración Agrícola China-América Latina y el Caribe por un monto de 50 millones de dólares, instaló el Programa de Asociación Científico-Tecnológica y el Programa de Intercambio entre Científicos Jóvenes China-América Latina y el Caribe, y organizó oportunamente el Primer Foro de Innovación Científico-Tecnológica entre China y América Latina y el Caribe. Todos los mecanismos de cooperación favorecen la ampliación de la magnitud del comercio y la inversión, y ofrecen un fuerte respaldo para la materialización de proyectos. La cooperación para el establecimiento de Zona de Libre Comercio ha resultado también muy fructífera. La implementación de los tratados de libre comercio que China firmó con Chile, Perú y Costa Rica han tenido una resultado satisfactorio. En noviembre del 2016, se anunció el arranque de las negociaciones sobre la actualización del TLC con Chile y el inicio del estudio conjunto sobre la actualización del TLC con Perú. Además, la parte china está explorando con la parte colombiana la factibilidad de efectuar estudios conjuntos para actualizar el tratado de libre comercio. El Gobierno uruguayo también ha planteado al gobierno chino, la idea de llevar a cabo cooperación sobre el establecimiento de zonas de libre comercio.

\section{c. En el ámbito cultural y humanístico}

El factor importante que obstaculiza un mayor desenvolvimiento de la cooperación entre ambos territorios es la falta de entendimiento mutuo como consecuencia de la distancia geográfica, las diferencias en cuanto a instituciones políticas, tradiciones, costumbres, lenguajes, así como las visiones distorsionadas de los hechos sobre China por parte de los medios occidentales. En el contexto general del ascenso de China y sus crecientes relaciones con América Latina, surgen opiniones negativas con respecto al "neocolonialismo" chino y la "Teoría de la Amenaza China".

Por este motivo, China busca promover el intercambio y la cooperación cultural con este continente, con el objetivo de profundizar dicho intercambio y el aprendizaje mutuos así como de mejorar su imagen. El 16 de julio de 2014, el presidente Xi Jinping pronunció el discurso en el congreso de Brasil y señaló que, el pueblo chino está luchando por la realización de la gran revitalización de la nación china. La unidad y la cooperación, el desarrollo y el renacimiento son sueños de generación a generación de los pueblos latinoamericanos. El sueño chino está íntimamente ligado con el sueño latinoamericano. Un día despúes, Xi Jinping asistió al Encuentro de los Líderes China-América Latina y el Caribe, y afirmó que China está dispuesta a fortalecer el intercambio con los gobiernos, los órganos legislativos, los partidos políticos y las localidades de los países latinoamericanos y caribeños y reforzar el intercambio y la cooperación en los terrenos como la educación, la cultura, el deporte, la prensa y el turismo. En los próximos cinco años, China proporcionará a los países de América Latina y el Caribe 6.000 becas del gobierno, 6.000 plazas para recibir capacitación en China así como 400 cupos de maestría a tiempo parcial. China invitará a 1.000 líderes de partidos políticos de los países latinoamericanos y caribeños para hacer visitas e intercambios en el país asiático, iniciará en el 2015 el programa "Un Puente hacia el Futuro" destinado a la formación de mil líderes jóvenes chinos y latinoamericanos. Asimismo, ha propuesto la celebración del "Año de Intercambio Cultural China-América Latina y el Caribe" en el 2016. En noviembre del mismo año como explicamos anteriormente- el gobierno chino publicó el segundo Documento sobre la Política de China hacia América Latina y el Caribe, en el cual se dio una mayor definición en el área cultural y humanística en comparación con el primer documento de este 
tipo. Se ha visto la importancia concedida a la cooperación en este terreno por parte del gobierno chino.

\section{d. En el ámbito de cooperación multilateral}

Con una mayor inserción de China en el sistema internacional, este país asiático impulsa el multilateralismo que se considera un mecanismo efectivo de preservar la paz y promover el desarrollo (Jinping, 2017). América Latina se esfuerza por desempeñar un papel más importante en los asuntos internacionales, y enfatiza la unidad a nivel regional (Creutzfeldt, 2016). En este sentido, la cooperación multilateral entre China y América Latina ha producido resultados fructíferos.

Se inició una nueva etapa de la Asociación de Cooperación Integral China-América Latina y el Caribe. Desde los años noventa del siglo XX, la cooperación multilateral entre ambas partes se implementó con las subregiónes de ALC, sin abarcar toda la región en su conjunto. El establecimiento de la Comunidad de Estados Latinoamericanos y del Caribe (CELAC) en 2011 permitió avances mayores en la cooperación en conjunto. Así fue que en enero de 2014, en la II Cumbre de CELAC se acordó la realización del Foro China-CELAC (FCC). Según mencionamos arriba, el 17 de julio de 2014, el Presidente de China, Xi Jinping, asistió a la reunión de líderes chino-latinoamericanos y caribeños en Brasilia, anunciando el establecimiento oficial del FCC y en enero de 2015 en la Primera Reunión Ministerial del FCC que tuvo lugar en Beijing, marcó el lanzamiento oficial del FCC y la cobertura completa de los mecanismos de cooperación integral en el terreno político, económico, cultural y de asuntos internacionales, con el fin de promover la construcción de "una comunidad de destino". El Presidente Solís de Costa Rica que ocupaba la presidencia pro tempore de CELAC, dijo que: "el FCC ha abierto un nuevo espacio para la expansión de la cooperación, ha extendido un nuevo puente para acrecentar la amistad entre sus pueblos y ha sentado las bases para el establecimiento de una relación de amistad duradera de las dos partes" ${ }^{\prime 5}$.

China y ALC se esfuerzan por profundizar las relaciones más integradas y una cooperación más coordinada en los mecanismos multilaterales internacionales. A partir del 2013, este país planteó la iniciativa de "una Franja y una Ruta", el Banco de Desarrollo de los BRICS y el Banco Asiático de Inversión en Infraestructura (BAll) que sirven para mejorar y complementar el orden económico internacional. Las naciones latinoamericanas juegan un papel activo en aquellos proyectos. Por ejemplo, Brasil es un miembro fundador del Banco de Desarrollo de los BRICS, y el único país de Latinoamérica que participó en el BAll desde el principio, seguido por Perú, Venezuela, Chile, Bolivia y Argentina, que fue aceptado como futuro miembro en mayo del 2017. Asimismo, otros países como Perú, Chile, Ecuador y Argentina apoyaron la iniciativa de "una Franja y una Ruta". Los presidentes de Argentina y Chile asistieron al Foro de "una Franja y una Ruta" para la Cooperación Internacional en Beijing.

5 http://news.china.com.cn/2015-01/08/content_34511668.htm, 访问日期：2017 年 7 月 1 日. Presidente de Costa Rica: el Foro China-CELAC favorece la financiación de infraestructura de América Latina. 


\section{Desafíos}

\section{a. Incertidumbre en el mundo}

Las Relaciones Internacionales contemporáneas se destacan por la "incertidumbre" y muchas veces por la dificultad de predecir ciertos acontecimientos con fuerte impacto a nivel global; tal es el caso de la salida de Gran Bretaña de la Unión Europea, del triunfo de Donald Trump o de los recientes ataques terroristas en Europa. Al mismo tiempo, la Comunidad Internacional todavía no ha encontrado una solución eficaz para resolver los problemas sobre la paz internacional, la seguridad regional, las disputas territoriales, el crecimiento económico, el libre comercio, la facilitación de la inversión y otras cuestiones políticas internacionales, el cambio climático y el deterioro del medio ambiente, entre otras. Estos fenómenos muestran que la humanidad está en un período de grandes cambios y se enfrenta a una serie de dilemas.

A pesar del escenario de incertidumbre mundial descripto, el desarrollo de las relaciones sino-latinoamericanas no se han visto afectadas. Muestra de ello lo constituye el hecho de que el 19 de mayo de 2016, el Ministro de Relaciones Exteriores de China, Wang Yi, y su homóloga Malcorra de Argentina, se reunieran junto con los medios de prensa para exponer la postura de China, respondiendo preguntas sobre si el cambio de la situación política de América Latina afectaba a las relaciones del país asiático con la región. En este sentido, Wang Yi, enfatizó sobre los tres "sin cambios": en primer lugar, la confianza de China en las perspectivas de desarrollo de América Latina no ha cambiado. En segundo lugar, se mantiene sin cambio la relación en la cual China y América Latina respectivamente se ofrecen oportunidades, se destacan ventajas complementarias económicas entre ellas y la cooperación mutua promueve efectivamente su desarrollo. En tercer lugar, la política de China para fortalecer la cooperación con los países de América Latina tampoco ha cambiado (Yi, 2017).

\section{b. Transformaciones complejas de China y América Latina}

No cabe dudas de que China ha experimentando transformaciones económicas en los últimos tiempos. Despúes de mantener un alto crecimiento durante varios años, el gobierno y la sociedad han tomado en cuenta que a pesar de ello, se producen muchos problemas, incluyendo la contaminación del aire, la inseguridad de los alimentos y la desigualdad del desarrollo social y económico, entre otros. El nuevo colectivo de liderazgo de China ha dedicado muchos esfuerzos para la reforma del modelo de desarrollo económico, a fin de evitar caer en la "trampa de ingreso medio". En la actualidad, China presta más atención a la prevención de riesgos económicos, para garantizar que no ocurran riesgos en el sistema financiero, y también enfatiza la coordinación de la política macroeconómica internacional con la idea de crear un entorno económico y comercial estable (Tong, 2017).

Los países de América Latina y el Caribe tienen muchas dificultades en sus transformaciones, afrontando un escenario desfavorable que se configuró por ejemplo en los efectos negativos de la crisis financiera internacional de 2008 y que se tradujo en: el populismo de derecha representado por Trump y la globalización desequilibrada. Este continente como proveedor de productos básicos al mercado internacional, se encuentra en un estado marginal, y ha sido gravemente afectado por la desaceleración económica 
mundial. La situación política no es tan estable, por lo que ni la izquierda ni la derecha cuentan con suficientes capacidades para realizar buenas gobernanzas, mostrando algunos signos de retroceso político. Las frecuentes crisis han dejado secuelas que se pueden explicar a través de los siguientes puntos: ineficiencias en el sistema democrático, problemas en el control a largo plazo por parte del poder hegemónico, la pérdida de la cultura tradicional, y la falta del nuevo concepto de desarrollo (Baiyi, 2016). Con el fin de superar los obstáculos, es clave que el gobierno, la sociedad y el pueblo lleguen a un consenso para hacer transformaciones sostenibles, y lograr con ello la capacidad de dialogar en forma igual con las potencias mundiales.

Las relaciones exteriores de las naciones latinoamericanas se encaminan hacia la diversidad, la multidireccionalidad y la multiplicidad, reflejándose en alternativas que pendulan entre el seguimiento a las potencias o la adhesión a la independencia, el enfoque del desarrollo económico o la ideología, y la protección comercial o el libre comercio. Frente a tantas diferencias que se pueden presentar entre ambas partes, China va buscando un terreno común y acepta la existencia de diferencias para profundizar el entendimiento mutuo.

\section{c. Varias contradicciones a ser resueltas}

En la actualidad, hay varias contradicciones importantes que deben ser resueltas: la primera, es la brecha entre el mejoramiento de mecanismos de cooperación y sus expectativas; la segunda, es la cada vez más fuerte presencia estratégica de China en América Latina y la capacidad de aceptación de este continente. La última es la necesidad urgente de desarrollar esta relación y la falta del conocimiento mutuo.

La influencia de China se ha extendido por el mundo. Debido a las diferencias en materia de historias, culturas, regímenes y tradiciones entre China y América Latina, se dificulta el entendimiento mutuo, y es inevitable que existan dudas, sospechas, e incluso discrepancias de ambos lados. Cabe destacar que el impacto de China en los países latinoamericanos no es totalmente positivo. En esta región surgen preocupaciones y dudas sobre las políticas y prácticas del país asiático y también se ha generado una opinión pública negativa sobre el mismo. Todo esto se puede explicar por tres razones: la primera es que América Latina está insatisfecha con la cooperación actual y tiene demasiadas expectativas de China. La segunda es la preocupación y las dudas sobre China, quejándose de que el modelo comercial entre ambos lados muestra una relación centro-periferia escondida en una Sur-Sur. La tercera es la falta de comprensión y confianza en China. Existen todavía imágenes tales como: "el nacionalismo de recursos" y "la amenaza de China". En julio de 2016, el presidente argentino Mauricio Macri asistió a la conferencia celebrada en Sun Valley, y señaló que "la inversión está viniendo de todas partes del mundo. Si todo viene de China, habrá un desequilibrio con el mundo. China ha sido emprendedora: está lista para poner todo el financiamiento e inversión en nuestras empresas. Pero necesitamos una situación de equilibrio en el país; pues tenemos una gran relación con Estados Unidos, que empezó con el viaje de (el presidente Barack) Obama y con la Unión Europea (es más fácil comerciar con Europa que con Asia) ${ }^{6}$ ". Por eso, China

6 Sun Valley conference: Argentine President Macri explains economic turnaround, http://www.cnbc.com/2016/07/07/sun-valley-conference-argentine-president-macri-explains-economicturnaround.html ( 
y América Latina deben conseguir un entendimiento mutuo para la cooperación y para la relación sustentada en la idea de ganar-ganar.

\section{Conclusión}

En resumen, después del XVIII Congreso Nacional del Partido Comunista de China, en el nuevo escenario que configura el ajuste de la estrategia diplomática del gobierno chino, las relaciones sino-latinoamericanas se han transformado del modelo reaccionario hacia la etapa de "desarrollo constructivo".

Durante esta transición, las dos partes se enfrentan a varios problemas y desafíos que quedan de manifiesto en sus estructuras comerciales y las diferencias en materia de la cultura, la historia, el régimen, el modelo de desarrollo y el valor, entre otros factores.

En definitiva, ambos lados deben impulsar sus lazos desde una mirada estratégica, planificar el concepto, la orientación y la ruta para el desarrollo y mejorar los conocimientos mutuos entre los pueblos. La confianza política mutua y la cooperación pragmática sirven como motor. La construcción del valor y del concepto cognitivo juega un papel fundamental para impulsar los vínculos y la cooperación por medio de un desarrollo sostenible, integral y profundo.

\section{Bibliografía}

Creutzfeldt, Benjamin (2016), "China and the U.S. in Latin America”, Revista Científica General José María Córdova, Bogotá, Colombia, enero-junio, Estudios militares - Vol. 14, Núm. 17, pp. 23-40.

Documento sobre la Política de China Hacia América Latina y el Caribe, http://www.fmprc.gov.cn/esp/wjdt/wjzc/t1418256.shtml, consultado el 24/11/2016.

Dussel Peters, Enrique - Armony, Ariel C. (2015) (coord.), Beyond Raw Materials: Who are the Actors in the Latin America and Caribbean-China Relationship? Buenos Aires : Nueva Sociedad ; Buenos Aires : Friedrich-Ebert-Stiftung ; México DF : Red Académica de América Latina y el Caribe sobre China ; Pittsburgh : University of Pittsburgh. Center of Latin American Studies, December

Baiyi, Wu (2016) Principales razones de la dificulta de Transformación de América Latina, Foro del pueblo, número 16.

Jiemian, Yang (2014) Realineamiento estratégico de la diplomacia china desde un punto nuevo, de la Revista Guojizhanwang, número 1

Shuangrong, He (2016), Historia de las relaciones entre China y los países latinoamericanos y caribeños, Editorial de Ciencias Sociales de China.

Shuangrong, He (2016) Historia de las relaciones entre China y los países latinoamericanos y caribeños, Editorial de Ciencias Sociales de China, 2016.

Hongying, Wu (2015): Relaciones sino-latinoamericanas están cambiando, de la revista Relaciones internacionales modernas, numero 5 de 2015. 
La nueva Etapa del "Desarrollo Constructivo" de las relaciones sino-latinoamericanas

Dingli, Shen (2016): Relaciones sino-latinoamericanas han entrado en el momento de gran cambio, Diario del Pueblo, versión de ultramar, 18 de noviembre de 2016

Baiyi, Wu (2015): Triple interpretación de la cooperación general entre China y América Latina, Revista: Inversión en China, número 2 de 2015.

${ }^{1}$ Latin American Economic Outlook (2016) Towards a New Partnership with China, http://www.oecd-

ilibrary.org/deliver/4115081e.pdf?itemld=/content/book/9789264246218-

en\&mimeType=application/pdf

Maoxiu, chen (S/F): China, país abierto desde hace 30 años, todavía no conoce nada de África y América Latina. http://blog.caijing.com.cn/mattferchen.

Maoxiu, Chen (2016) China, país abierto desde hace 30 años, todavía no conoce nada de África y América Latina.

Textos de los discursos del Presidente Xi Jinping(2016), Departamento de Publicidad del Partido Comunista de China, Editorial de Xuexi, Editorial del Pueblo, abril de 2016

Jiemian, Yang (2014), Realineamiento estratégico de la diplomacia china desde un punto nuevo, de la Revista Guojizhanwang, número 1 de 2014

Jiemian, Yang (2014) Realineamiento estratégico de la diplomacia china desde un punto nuevo, de la Revista Guojizhanwang, número 1 de 2014

Yi, Wang (2017) Establecer la asociación y lograr un desarrollo pacífico conjuntamente, Periódico de aprendizaje, 29 de marzo de 2017.

Página web de Ministerio de Comercio, fecha de consulta:10 de abril de 2017 Disponible en http://www.mofcom.gov.cn/article/ae/ai/201702/20170202513555.shtml.

A golden opportunity : China's president ventures into Donald Trump's backyard, https://www.economist.com/news/americas/21710307-chinas-presidentventures-donald-trumps-backyard-golden-opportunity

Jinping, Xi (2017), Trabajen juntos para construir una comunidad de futuro compartido para la humanidad-- discurso en la Oficina de Naciones Unidas en Ginebra, Diario de Pueblo, 20 de enero de 2017.

http://news.china.com.cn/2015-01/08/content_34511668.htm, 访问日期：2017年7月1日.

Presidente de Costa Rica: el Foro China-CELAC favorece la financiación de infraestructura de América Latina.

${ }^{1}$ 王毅谈中拉矢系：三个“没有变”，http://www.fmprc.gov.cn/web/wjbzhd/t1364828.shtml

Tong, Xiong (2017) : la otra mitad de la tendencia de la economía global y la transformación económica de China; entrevista especial a Li WEI, director del Centro de Investigación de desarrollo del Consejo de Estado, HUANQIU, número 17 de 2017.

Baiy, wu (2016)i: Principales razones de la dificultad de Transformación de América Latina, Foro del pueblo, número 16 de 2016.

Sun Valley conference: Argentine President Macri explains economic turnaround, http://www.cnbc.com/2016/07/07/sun-valley-conference-argentinepresident-macri-explains-economic-turnaround.html 